\title{
Dynamic analysis of the stabilized meshfree method for the Mindlin-Reissner plate
}

\author{
Dongdong Wang \& Yiling Zhang \\ Department of Civil Engineering, Xiamen University, China
}

\begin{abstract}
A dynamic analysis of the stabilized conforming nodal integration meshfree Mindlin-Reissner plate formulation is carried out and compared with the meshfree approach using Gauss integration. The dispersion performance is discussed with particular reference to the locking-free characteristic of the semidiscrete formulation. Subsequently, the space-time fully discretized meshfree equations are established by further invoking the Newmark time integration method which is followed by a transient analysis. The numerical results of both dispersion and transient analyses demonstrate that the meshfree formulation based on the conventional Gauss integration shows severe locking problems while the stabilized conforming nodal integration meshfree plate formulation is locking-free and has very high accuracy.

Keywords: meshfree method, stabilized conforming nodal integration, MindlinReissner plate, locking, dynamic analysis.
\end{abstract}

\section{Introduction}

Meshfree methods employ particle-based discretization which provides great flexibility for the construction of conforming and high order smoothing approximations, adaptive local refinement, moving boundary modelling, and large deformation analysis. Thus, during the past twenty years these classes of methods experienced very rapid development [1]. In this study, we refer to the moving least square or reproducing kernel based Galerkin meshfree method [2, $3]$. The computational efficiency is crucial for the application of this type of method to large scale problems. A direct approach which has high efficiency is the nodal integration Galerkin meshfree method. However, this method suffers from the rank instability problem $[4,5]$. Various stabilization techniques have 
been proposed to overcome the rank deficiency within the nodal integration framework. Beissl and Belytscko [4] proposed the residual stabilization method with a discretization-dependent controlling parameter. Chen et al. [5] presented a general strain smoothing framework and developed a linearly exact stabilized conforming nodal integration (SCNI) Galerkin meshfree method without artificial parameter. As for the structural problems, Wang and Chen [6] introduced a bending-exact locking-free SCNI formulation for the MindlinReissner plate. Subsequently, this method was further developed for shell problems [7]. More recently a sub-domain stabilized conforming integration method was also systematically developed for Galerkin meshfree analysis of thin beam and plate structures [8].

The static locking-free behaviors of the SCNI meshfree Mindlin-Reissner plate formulation have been investigated in [6]. In this work, the dynamic performance of this approach is examined and compared with the Galerkin meshfree formulation using 5 by 5 Gauss integration. The mass contribution is evaluated by nodal integration within the nodal representative domain. Firstly, the accuracy of the semi-discrete meshfree equations is studied by the dispersion analysis. The space-time fully discrete meshfree equations for the MindlinReissner plate are formulated by further employing the Newmark time integration method and thereafter the transient analysis is carried out. The dispersion and transient results are presented to assess the dynamic accuracy of the proposed SCNI meshfree formulation for the Mindlin-Reissner plate.

\section{Meshfree approximation theory}

Consider a problem domain $\bar{\Omega}=\Omega \cup \Gamma$, where $\Omega$ and $\Gamma$ denote the interior domain and boundary, respectively. In the moving least squares and reproducing kernel meshfree approximation, $\bar{\Omega}$ is represented by a set of particles $\mathbf{x}_{I}$, $I=1,2, \ldots, N P$. The meshfree approximant of a filed variable, say, the plate deflection $w(\mathbf{x}, t)$, denoted by $w^{h}(\mathbf{x}, t)$, can be expressed as [3]:

$$
w^{h}(\mathbf{x}, t)=\sum_{I=1}^{N P} \Psi_{I}(\mathbf{x}) w_{I}(t)
$$

where $\Psi_{I}(\mathbf{x})$ is the meshfree shape function associated with the particle $\mathbf{x}_{I}$, and $w_{I}(t)$ is the time dependent nodal coefficient. Generally, $\Psi_{I}(\mathbf{x})$ takes the following form [3]:

$$
\Psi_{I}(\mathbf{x})=\mathbf{p}^{T}\left(\mathbf{x}_{I}-\mathbf{x}\right) \mathbf{a}(\mathbf{x}) \phi_{a}\left(\mathbf{x}_{I}-\mathbf{x}\right),
$$

where $\phi_{a}\left(\mathbf{x}_{I}-\mathbf{x}\right)$ is the cubic B-spline basis function with a local support size of " $a$ " [1]. $\mathbf{p}(\mathbf{x})$ is the $n$-th order monomial kernel vector:

$$
\mathbf{p}(\mathbf{x})=\left\{1, x, y, x^{2}, x y, y^{2}, \ldots, x^{n}, \ldots, y^{n}\right\}^{T} .
$$


The unknown coefficient vector $\mathbf{a}(\mathbf{x})$ is derived from the following consistency conditions:

$$
\sum_{I=1}^{N P} \Psi_{I}(\mathbf{x}) \mathbf{p}\left(\mathbf{x}_{I}-\mathbf{x}\right)=\mathbf{p}(\mathbf{0}),
$$

Substituting Eq. (2) into (4) gives:

$$
\mathbf{a}(\mathbf{x})=\mathbf{A}^{-1}(\mathbf{x}) \mathbf{p}(\mathbf{0}), \mathbf{A}(\mathbf{x})=\sum_{I=1}^{N P} \mathbf{p}^{T}\left(\mathbf{x}_{I}-\mathbf{x}\right) \mathbf{p}\left(\mathbf{x}_{I}-\mathbf{x}\right) \phi_{a}\left(\mathbf{x}_{I}-\mathbf{x}\right),
$$

where $\mathbf{A}(\mathbf{x})$ is the moment matrix:

$$
\mathbf{A}(\mathbf{x})=\sum_{I=1}^{N P} \mathbf{p}^{T}\left(\mathbf{x}_{I}-\mathbf{x}\right) \mathbf{p}\left(\mathbf{x}_{I}-\mathbf{x}\right) \phi_{a}\left(\mathbf{x}_{I}-\mathbf{x}\right) .
$$

Consequently the meshfree shape function is given by:

$$
\Psi_{I}(\mathbf{x})=\mathbf{p}^{T}(\mathbf{0}) \mathbf{A}^{-1}(\mathbf{x}) \mathbf{p}\left(\mathbf{x}_{I}-\mathbf{x}\right) \phi_{a}\left(\mathbf{x}_{I}-\mathbf{x}\right) .
$$

\section{SCNI meshfree plate formulation}

\subsection{Basic plate equations}

According to the Mindlin-Reissner plate theory, the primary variables at a point $\mathbf{x}$ on the plate mid-surface $\Omega$ are the deflection $w(\mathbf{x}, t)$ and the rotations $\theta_{x}(\mathbf{x}, t)$ and $\theta_{y}(\mathbf{x}, t)$ as shown in Fig. 1. Accordingly the curvature and shear strain vectors $\boldsymbol{\kappa}$ and $\boldsymbol{\gamma}$ are:

$$
\boldsymbol{\kappa}=\left\{\begin{array}{c}
\kappa_{x x} \\
\kappa_{y y} \\
2 \kappa_{x y}
\end{array}\right\}=\left\{\begin{array}{c}
\frac{\partial \theta_{x}}{\partial x} \\
\frac{\partial \theta_{y}}{\partial y} \\
\frac{\partial \theta_{x}}{\partial y}+\frac{\partial \theta_{y}}{\partial x}
\end{array}\right\}, \boldsymbol{\gamma}=\left\{\begin{array}{l}
\gamma_{x} \\
\gamma_{y}
\end{array}\right\}=\left\{\begin{array}{l}
\frac{\partial w}{\partial x}-\theta_{x} \\
\frac{\partial w}{\partial y}-\theta_{y}
\end{array}\right\}
$$

The constitutive equations that relate the moment vector $\mathbf{m}$ and shear force vector $\mathbf{s}$ with the curvature and shear strain vectors read:

$$
\mathbf{m}=\left\{\begin{array}{l}
m_{x x} \\
m_{y y} \\
m_{x y}
\end{array}\right\}=-\mathbf{D}^{b} \mathbf{\kappa}, \mathbf{s}=\left\{\begin{array}{l}
s_{x} \\
s_{y}
\end{array}\right\}=\mathbf{D}^{s} \gamma,
$$

with 
96 Boundary Elements and Other Mesh Reduction Methods XXXVI

$$
\mathbf{D}^{b}=\frac{E h^{3}}{12\left(1-v^{2}\right)}\left[\begin{array}{ccc}
1 & v & 0 \\
v & 1 & 0 \\
0 & 0 & (1-v) / 2
\end{array}\right], \boldsymbol{D}^{s}=k_{s} h G\left[\begin{array}{ll}
1 & 0 \\
0 & 1
\end{array}\right],
$$

where $E$ and $v$ are the Young's modulus and Poisson's ratio, $h$ is the plate thickness, $k_{s}$ is the shear correction factor and taken as $5 / 6$ herein, $G$ is the shear modulus.

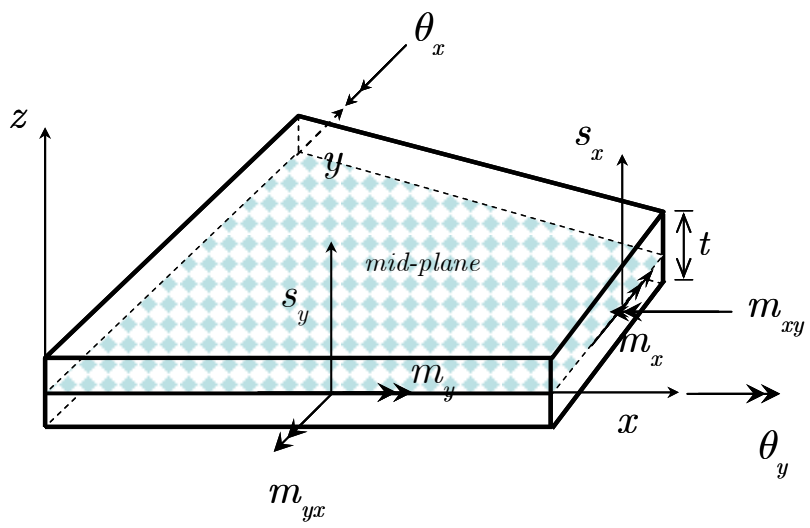

Figure 1: $\quad$ Schematic illustration of plate sign conventions.

The governing equation for plate motion is:

$$
\left\{\begin{array}{l}
\rho I \frac{\partial^{2} \theta_{x}}{\partial t^{2}}=\frac{\partial m_{x x}}{\partial x}+\frac{\partial m_{x y}}{\partial y}-s_{x}, \\
\rho I \frac{\partial^{2} \theta_{y}}{\partial t^{2}}=\frac{\partial m_{x y}}{\partial x}+\frac{\partial m_{y x}}{\partial y}-s_{y}, \\
\rho h \frac{\partial^{2} w}{\partial t^{2}}=\frac{\partial s_{x}}{\partial x}+\frac{\partial s_{y}}{\partial y}+q,
\end{array}\right.
$$

where $I=h^{3} / 12, \rho$ is the density per unit mid-surface area, $q$ is the applied transverse loading. The corresponding weak formulation of Eq. (11) is

$$
\begin{aligned}
& \int_{\Omega}\left(\delta w \rho h \ddot{w}+\delta \theta_{x} \rho I \ddot{\theta}_{x}+\delta \theta_{x} \rho I \ddot{\theta}_{y}\right) d \Omega \\
& +\int_{\Omega} \delta \boldsymbol{\kappa}^{T} \boldsymbol{D}^{b} \boldsymbol{\kappa} d \Omega+\int_{\Omega} \delta \boldsymbol{\gamma}^{T} \boldsymbol{D}^{s} \gamma d \Omega-\delta W^{e x t}=0
\end{aligned}
$$

in which $\delta W^{e x t}$ is the virtual work done by external forces. 


\subsection{SCNI discrete formulation}

In the SCNI formulation, to achieve the bending exactness and spatial stability, a smoothed curvature $\tilde{\mathbf{\kappa}}$ within a nodal representative domain $\Omega_{K}$ as shown in Fig. 2 is constructed as follows [6]:

$$
\tilde{\mathbf{\kappa}}(\mathbf{x}, t)=\left\{\begin{array}{c}
\tilde{\kappa}_{x x} \\
\tilde{\boldsymbol{\kappa}}_{y y} \\
2 \tilde{\kappa}_{x y}
\end{array}\right\}=\frac{1}{A_{K}} \int_{\Omega_{K}}\left\{\begin{array}{c}
\kappa_{x x} \\
\kappa_{y y} \\
2 \kappa_{x y}
\end{array}\right\} d \Omega=\frac{1}{A_{K}} \int_{\Gamma_{K}}\left\{\begin{array}{c}
\theta_{x} n_{x} \\
\theta_{y} n_{y} \\
2 \theta_{x} n_{y}
\end{array}\right\} d \Omega
$$

where the divergence theorem is used, $\Gamma_{K}$ and $A_{K}$ are the boundary and area of $\Omega_{K}, \mathbf{n}=\left\{n_{x}, n_{y}\right\}^{T}$ is the outward normal of $\Gamma_{K}$.

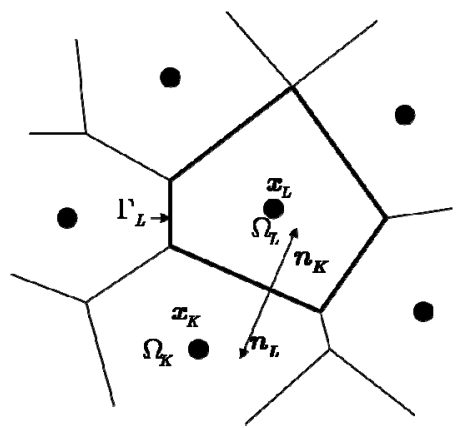

Figure 2: $\quad$ Nodal representative domain.

We invoke the following meshfree approximation of the primary variables:

$$
\mathbf{u}^{h}(\mathbf{x}, t)=\left\{\begin{array}{c}
w^{h}(\mathbf{x}, t) \\
\theta_{x}^{h}(\mathbf{x}, t) \\
\theta_{y}^{h}(\mathbf{x}, t)
\end{array}\right\}=\sum_{I=1}^{N P}\left[\begin{array}{ccc}
\Psi_{I}(\mathbf{x}) & 0 & 0 \\
0 & \Psi_{I}(\mathbf{x}) & 0 \\
0 & 0 & \Psi_{I}(\mathbf{x})
\end{array}\right] \mathbf{d}_{I}(t)=\Psi(\mathbf{x}) \mathbf{d}(t)
$$

where $\mathbf{d}_{I}=\left\{\begin{array}{lll}w_{I} & \theta_{x I} & \theta_{y I}\end{array}\right\}^{T}$. Substituting Eq. (14) into Eq. (13) gives the discrete smoothed curvature:

$$
\tilde{\mathbf{\kappa}}^{h}\left(\mathbf{x}_{K}\right)=\sum_{I=1}^{N P} \tilde{\mathbf{B}}_{I}^{b}\left(\mathbf{x}_{K}\right) \mathbf{d}_{I}, \tilde{\mathbf{B}}_{I}^{b}\left(\mathbf{x}_{K}\right)=\left[\begin{array}{ccc}
0 & \tilde{\nabla}_{x} \Psi_{I}\left(\mathbf{x}_{K}\right) & 0 \\
0 & 0 & \tilde{\nabla}_{y} \Psi_{I}\left(\mathbf{x}_{K}\right) \\
0 & \tilde{\nabla}_{y} \Psi_{I}\left(\mathbf{x}_{K}\right) & \tilde{\nabla}_{x} \Psi_{I}\left(\mathbf{x}_{K}\right)
\end{array}\right],
$$

in which $\tilde{\nabla}_{\alpha} \Psi_{I}\left(\mathbf{x}_{K}\right)$ is given by: 


$$
\tilde{\nabla}_{\alpha} \Psi_{I}\left(\mathbf{x}_{K}\right)=\frac{1}{A_{K}} \int_{\Gamma_{K}} \Psi_{I}(\mathbf{x}) n_{\alpha}(\mathbf{x}) d \Gamma, \alpha=\{x, y\} .
$$

A nodal integration using the smoothed curvature measure of Eq. (15) for the weak form of Eq. (12) gives the semi-discrete equation:

$$
\mathbf{M d}+\mathbf{K d}=\mathbf{f},
$$

where

$$
\begin{aligned}
& \left\{\begin{array}{l}
\mathbf{M}_{I J}=\sum_{K=1}^{N P} \boldsymbol{\Psi}_{I}^{T}\left(\mathbf{x}_{K}\right) \operatorname{diag}[\rho h, \rho I, \rho I] \boldsymbol{\Psi}_{J}\left(\mathbf{x}_{K}\right) A_{K}, \\
\mathbf{K}_{I J}^{b}=\sum_{K=1}^{N P} \tilde{\mathbf{B}}_{I}^{b}\left(\mathbf{x}_{K}\right) \mathbf{D}^{b} \tilde{\mathbf{B}}_{J}^{b}\left(\mathbf{x}_{K}\right) A_{K}, \mathbf{K}_{I J}^{s}=\sum_{K=1}^{N P} \mathbf{B}_{I}^{s}\left(\mathbf{x}_{K}\right) \mathbf{D}^{s} \mathbf{B}_{J}^{s}\left(\mathbf{x}_{K}\right) A_{K},
\end{array}\right. \\
& \mathbf{f}_{I}=\sum_{K=1}^{N P} \boldsymbol{\Psi}_{I}\left(\mathbf{x}_{K}\right) \operatorname{diag}\left[0,0, q\left(\mathbf{x}_{K}\right)\right] A_{K}, \mathbf{B}^{s}=\left[\begin{array}{ccc}
\Psi_{I, x} & -\Psi_{I} & 0 \\
\Psi_{I, y} & 0 & -\Psi_{I}
\end{array}\right] .
\end{aligned}
$$

The homogeneous form of Eq. (17), i.e., $\mathbf{M} \ddot{\mathbf{d}}+\mathbf{K d}=\mathbf{0}$, is often used in dispersion analysis in order to assess the semi-discretization accuracy. In dispersion analysis, the nodal coefficient vector $\mathbf{d}_{I}=\mathbf{d}_{(i, j)}$ is assumed to take the following form [8]:

$$
\mathbf{d}_{(i, j)}(t)=\left\{\begin{array}{l}
w_{0} \exp \left[l\left(k x_{i} \cos \theta+k y_{j} \sin \theta-\omega^{h} t\right)\right], \\
\theta_{x 0} \exp \left[l\left(k x_{i} \cos \theta+k y_{j} \sin \theta-\omega^{h} t\right)\right], \\
\theta_{y 0} \exp \left[\imath\left(k x_{i} \cos \theta+k y_{j} \sin \theta-\omega^{h} t\right)\right],
\end{array}\right.
$$

where $\imath=\sqrt{-1}, w_{0}, \theta_{x 0}, \theta_{y 0}$ are the wave amplitudes, $k$ is the wave number, $\theta$ is the angle between the wave propagation direction and the $x$-axis, and $\omega^{h}$ is the numerical frequency to be determined by substituting Eq. (20) into the homogenous semi-discrete equation. After we obtain $\omega^{h}$, the normalized phase speed $c_{n}=\omega^{h} / \omega$ can be used to measure the accuracy of the proposed algorithm.

We next introduce the Newmark time discretization:

$$
\left\{\begin{array}{l}
\hat{\mathbf{d}}_{n+1}=\mathbf{d}_{n}+\Delta t \dot{\mathbf{d}}_{n}+\frac{\Delta t^{2}}{2}(1-2 \beta) \ddot{\mathbf{d}}_{n}, \hat{\dot{\mathbf{d}}}_{n+1}=\dot{\mathbf{d}}_{n}+\Delta t(1-\vartheta) \ddot{\mathbf{d}}_{n}, \\
\mathbf{d}_{n+1}=\hat{\mathbf{d}}_{n+1}+\beta \Delta t^{2} \ddot{\mathbf{d}}_{n+1}, \dot{\mathbf{d}}_{n+1}=\hat{\dot{\mathbf{d}}}_{n+1}+\vartheta \Delta t \ddot{\mathbf{d}}_{n+1},
\end{array}\right.
$$

where $\beta$ and $\vartheta$ are parameters, the subscripts $n$ and $(n+1)$ denote the time steps $t_{n}$ and $t_{n+1}$, and $\Delta t=t_{n+1}-t_{n}$. Thus the full discrete equation for the plate motion becomes: 


$$
\left(\mathbf{M}+\beta \Delta t^{2} \mathbf{K}\right) \ddot{\mathbf{d}}_{n+1}=\mathbf{f}_{n+1}-\mathbf{K} \hat{\mathbf{d}}_{n+1} .
$$

In the case that $\beta=0, \vartheta=1 / 2$, the Newmark integration method represents the central difference scheme.

\section{Numerical results}

\subsection{Dispersion analysis results}

Consider a square plate whose geometry and material properties are: length $L=10$, density $\rho=8000$, Young's modulus $E=2 \times 10^{11}$, and Poisson ratio $v=0.3$. To test the locking performance, the plate thickness is varied from 1 to 0.002 that correspond to the length/thickness ratios ranging from 10 to 5000 . This plate is uniformly discretized with 17 by 17 particles. A quadratic basis and a cubic B-spline kernel function with a normalized support size of 2.5 are employed in the meshfree approximation. Figures 3-4 show the normalized phase speed for the meshfree formulations using 5 by 5 Gauss integration and the proposed SCNI approach, respectively. The results clearly demonstrate that the proposed SCNI method yields very accurate solutions and is insensitive to the length/thickness ratio, which demonstrate its locking-free characteristic. On the other hand, as shown in Fig. 4 the results by 5 by 5 Gauss integration exhibit severe shear locking as the length/thickness ratio becomes large.

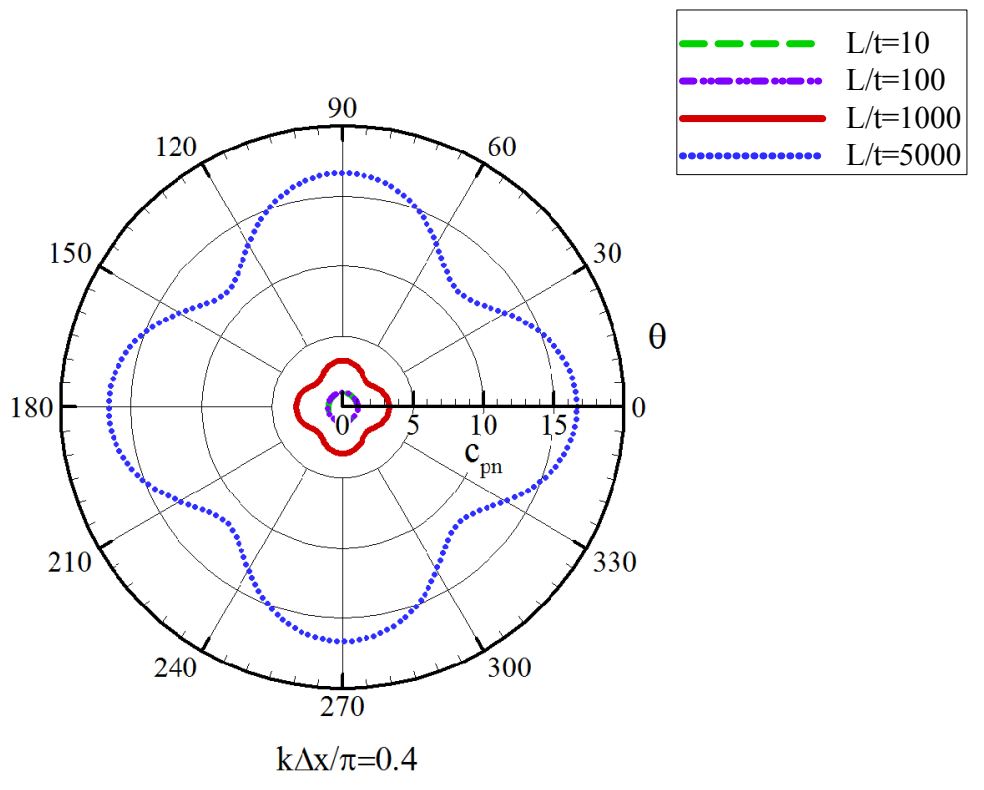

Figure 3: Normalized phase speed results with 5 by 5 Gauss integration meshfree formulation. 


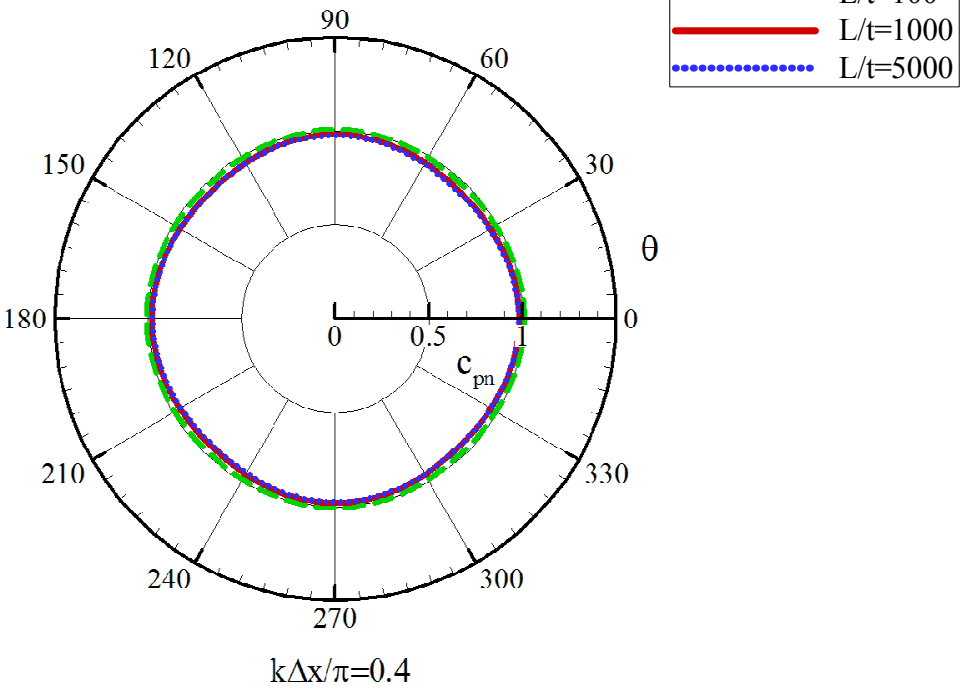

Figure 4: $\quad$ Normalized phase speed results by SCNI meshfree formulation.

\subsection{Dispersion analysis results}

Consider a simply supported square plate with the geometry and material parameters given by: length $L=10$, thickness $t=0.01$, density $\rho=8000$, Young's modulus $E=2 \times 10^{11}$, Poisson ratio $v=0.3$. This plate is subjected to a harmonic point load $F=100 \sin (\pi t)$ at the center. In the dynamic analysis a uniform discretization of 9 by 9 meshfree particles is employed. The meshfree approximation is constructed based on a quadratic basis function and a cubic Bspline kernel function with a normalized support size of 3 . The time step for the central difference time integration is $\Delta t=0.01$. Figure 5 shows the time history curve for the plate center using the meshfree formulation with 5 by 5 Gauss quadrature, where due to the shear locking, the plate deflection is clearly underestimated. The results in Fig. 6 instead show that the plate center deflection by the present SCNI method matches very well with the reference solution and no shear locking occurs. 


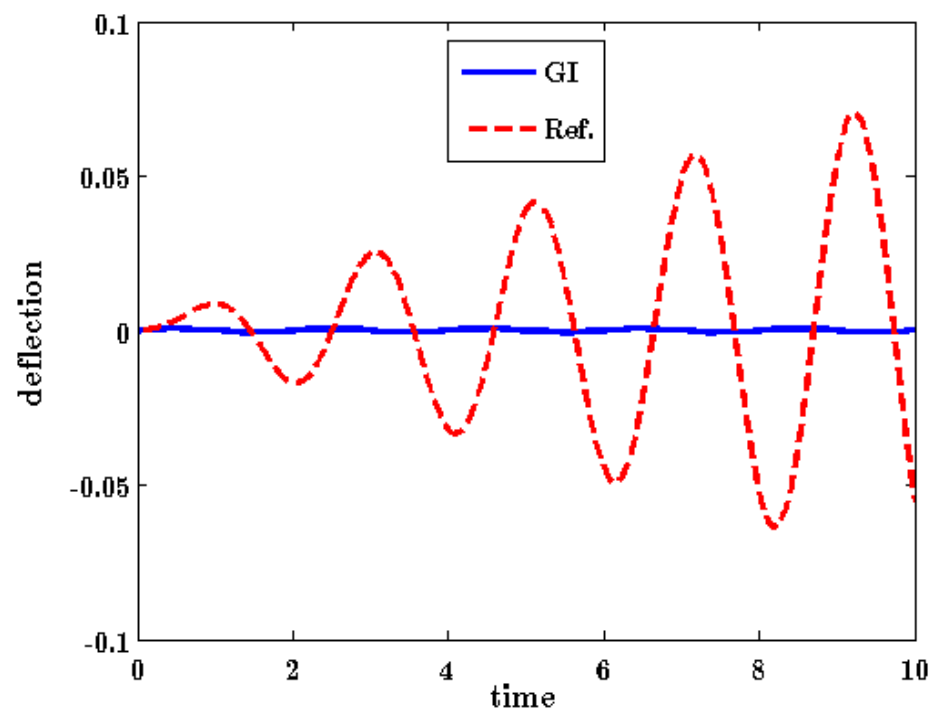

Figure 5: Time history curve of the plate center by the meshfree formulation with 5 by 5 quadrature.

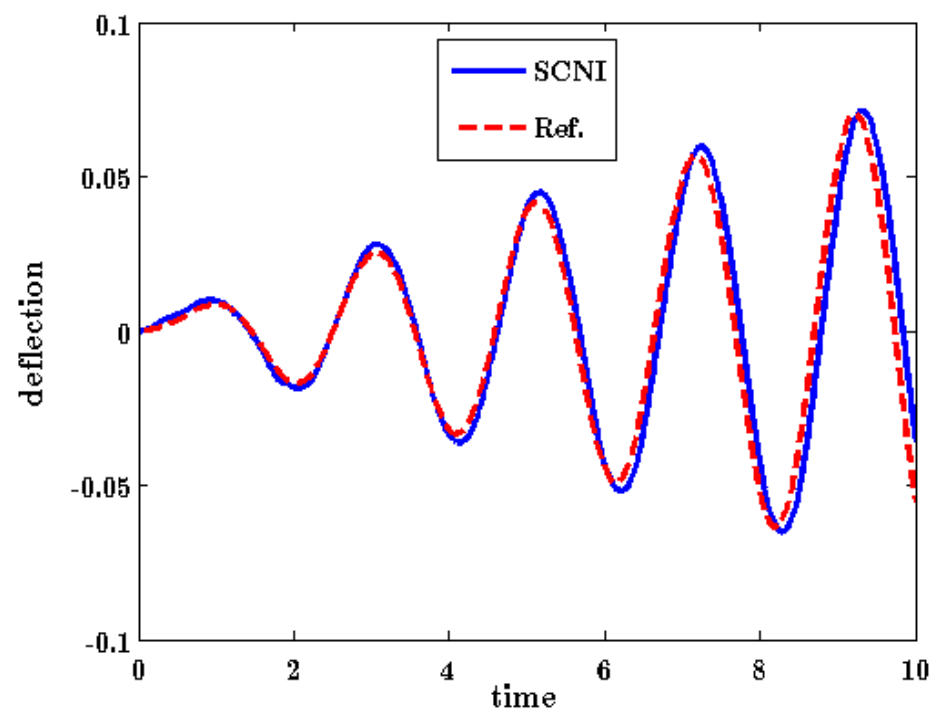

Figure 6: Time history curve of the plate center by the SCNI method. 


\section{Summary}

The dynamic behavior of the stabilized conforming nodal integration meshfree Mindlin-Reissner plate formulation has been investigated. The proposed method employs the smoothed curvature to meet the stability requirement in a nodal integration fashion. The semi- and full- discrete meshfree equations were presented. Based on the semi-discretization, the dispersion analysis was performed and the results show that the present method is locking-free and is very accurate. In contrast, the 5 by 5 Gauss quadrature meshfree formulation has severe shear locking. With the full-discretization, the transient analysis results once again confirm the locking issue associated with the 5 by 5 Gauss integration meshfree formulation as well as the locking-free property of the present SCNI approach.

\section{Acknowledgement}

The support of this work by the National Natural Science Foundation of China (11222221) is gratefully acknowledged.

\section{References}

[1] Liu, G.R., Meshfree Methods: Moving Beyond the Finite Element Method (2nd edition). CRC Press: Singapore, 2009.

[2] Belytschko, T., Lu, Y.Y. and Gu, L. Element free Galerkin methods. International Journal for Numerical Methods in Engineering, 37, pp. 229256, 1994.

[3] Liu, W.K., Jun, S. and Zhang, Y.F, Reproducing kernel particle methods. International Journal for Numerical Methods in Fluids, 20, pp. 1081-1106, 1995.

[4] Beissl, S. and Belytschko, T., Nodal integration of the element-free Galerkin method. Computer Methods in Applied Mechanics and Engineering, 139, pp. 49-64, 1996.

[5] Chen, J.S., Wu, C.T., Yoon, S. and You, Y. A stabilized conforming nodal integration for Galerkin meshfree methods. International Journal for Numerical Methods in Engineering, 50, pp. 435-466, 2001.

[6] Wang, D. and Chen, J.S., Locking-free stabilized conforming nodal integration for meshfree Mindlin-Reissner plate formulation. Computer Methods in Applied mechanics and Engineering, 193, pp. 1065-1083, 2004.

[7] Chen, J.S., and Wang, D., A constrained reproducing kernel particle formulation for shear deformable shell in Cartesian coordinates. International Journal for Numerical Methods in Engineering, 68, pp. 151$172,2006$.

[8] Wang, D. and Lin, Z. Dispersion and transient analyses of Hermite reproducing kernel Galerkin meshfree method with sub-domain stabilized conforming integration for thin beam and plate structures. Computational Mechanics, 48, pp. 47-63, 2011. 